\title{
Corrugation of the skull in Paget's disease of bone
}

\author{
N. K. Chakravorty \\ M.B.B.S., M.R.C.P.(E), D.T.M. \& H. \\ S. K. DAS* \\ M.B., M.R.C.P. \\ M. S. KATARIA $\uparrow$ \\ M.R.C.S., L.R.C.P., D.P.H., D.I.H. \\ St Luke's Hospital, Crosland Moor, Huddersfield, *Manor Hospital, Uttoxeter Road, \\ Derby, and †St Francis' Hospital, St Francis' Road, King's College Hospital Group, London
}

\begin{abstract}
Summary
Paget's disease of bone has been known for about 100 years and the usual deformities of bone, e.g. bowed tibia, large head, are well described in medical text books. However, there does not appear to have been a description of corrugation of the skull as a recognized deformity in Paget's disease. Three cases are now described to illustrate this deformity as an unusual but valuable sign in this disease.
\end{abstract}

\section{Introduction}

Paget's disease (osteitis deformans) affects 3-4\% of people of West European extraction aged over 55 years and perhaps $10 \%$ of the very old. In perhaps $5 \%$ of patients the disease is associated with clinically important symptoms (Leading Article, 1975). Paget's disease is often asymptomatic and is found incidentally during radiological examination of the skeleton carried out for other reasons (Hodkinson, 1974). The disease is most often recognized clinically because of bone enlargement and deformity of skull, femora, tibiae, as evident from a large, warm head, bitemporal enlargement of the skull, presence of bruit on auscultation of head (because of greatly increased vascularity) and bowing of legs, etc. Radiological changes in Paget's disease are characterized by destruction of bone followed by reparative changes. The destructive phase may predominate but most frequently there is a combination of destruction and repair, with expansion of the bone. The destructive phase is most often seen in the skull when it is known as 'osteoporosis circumscripta'. Characteristically large, well circumscribed areas of decalcification are seen. This most commonly involves the frontal and basal regions (Radiological Atlas). A commoner manifestation of Paget's dis-

Requests for reprints: Dr N. K. Chakravorty, St Luke's Hospital, Crosland Moor, Huddersfield HD4 5RQ. ease is new bone formation. This leads to sclerosis, expansion of bone and coarsening of trabeculae. The new bone is laid down in a haphazard fashion thus disturbing the normal bony architecture. In the skull, changes begin in the outer table. At first, islands of dense bone are seen, later differentiation between inner and outer tables is lost (Collins, 1956) and the skull may be thickened from two to five times its normal size (Sutton, 1975). Paget's disease may cause headache. The bone softening may lead to basilar invagination (Barry, 1969). Deformity is frequently seen in the weight-bearing bones, e.g. tibia and femur, with anterior and lateral bowing. Deformation of affected bones may cause painful osteoarthritis of the hip or knee. The pelvis may be compressed, leading to 'protrusio acetabuli' and a triradiate deformity (Fig. 5).

However, a corrugated and wavy pattern of the skull (Figs 2 and 3 ) in Paget's disease of bone, is a feature which has only recently been recognized (Das and Kataria, 1975a). The other associated features are deafness, congestive cardiac failure (high output failure) (Edholm, Howarth and McMichael, 1945), platybasia (flattening of the base of the skull). The disease may affect only one bone or part of a bone or may spread to involve a major part of the skeleton. The sacrum and pelvis are most commonly involved, followed closely by the femur and tibia (Beeson and McDermott, 1968). Less frequently involved bones are lumbar, thoracic and cervical vertebrae. The skull is affected in a significant proportion $(20 \%)$ of cases. Bones of upper limb and face are less often affected. The disease is characterized by extremely rapid bone formation and resorption in affected regions. The repair is achieved by deposition of primitive and highly abnormal bone. There is chaotic remodelling of bone sometimes resulting in an undulation of the 
skull bones, which can be seen and felt as corrugation of the skull. The replacement bone is architecturally and chemically abnormal. It is unstable and has little mechanical significance: fracture of long bones occurs in $30 \%$ of cases. Minor degree of deformity of the base of skull does not give rise to symptoms. With more severe invagination of the cervical spine into the base of the posterior fossa of the skull, there may be signs of impaired function of pyramidal tracts, posterior column and function of the cerebellum. Bone overgrowth may lead to foraminal encroachment which commonly causes deafness to develop, and rarely to optic atrophy.

Serum alkaline phosphatase is usually raised. It may reach very high levels (Boyd, 1970) when the disease is active or when an osteosarcoma is complicating Paget's disease. Excretion of urinary hydroxyproline is also increased as a result of the increased bone breakdown; the serum calcium level is unaffected unless the patient is immobilized owing to fracture of the femur. The serum phosphorus level is usually normal.

\section{Case 1}

A 92-year-old man presented with a 30-year history of Paget's disease with deformity of lower limbs (bowing) and skull (corrugation), (Fig. 1). He had complained of aching pain in the lower and

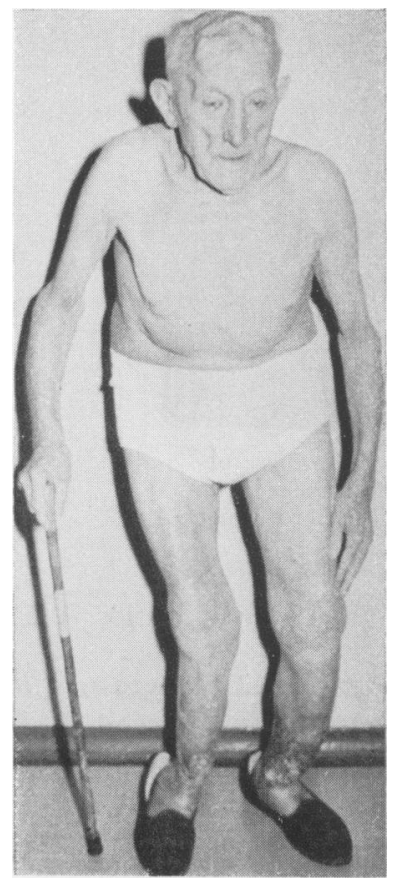

Fig. 1. Case no. 1. Photograph showing wavy head (gross corrugation of the skull) and the deformities of the legs, etc., due to Paget's disease.

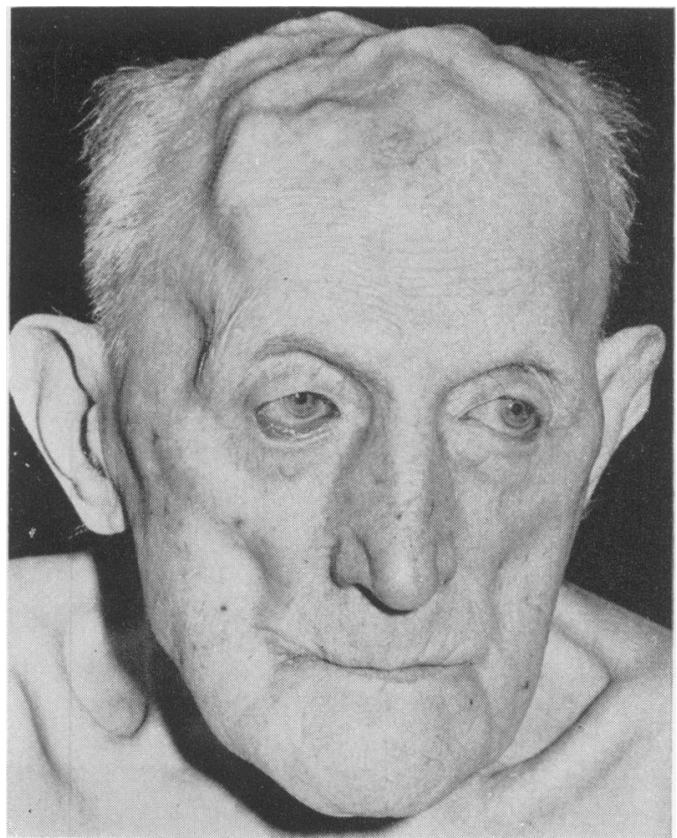

Fig. 2. Case no. 1. Closer view showing marked wavy pattern of the head (skull).

upper limbs for many years. He had had some swelling of feet for a few years (controlled by diuretic therapy). He had undergone prostatectomy 18 years previously for a benign lesion (proved histologically). He was partially sighted. He walked quite well with the help of a stick. There was no family history of Paget's disease.

The patient was very deaf but had no evidence of mental impairment. His blood pressure was $140 / 80$ $\mathrm{mmHg}$. There was cardiomegaly and a systolic murmur was heard in the aortic area. There was atrial fibrillation with moderate congestive cardiac failure (on diuretics). Examination of the chest showed gross kyphosis and evidence of emphysema. Examination of the nervous system revealed no other evidence of neurological deficit, apart from deafness and visual impairment. Abdominal examination was non-contributory.

\section{Investigations}

Haemoglobin, $14.3 \mathrm{~g} / \mathrm{dl}$; white blood cells, $4000 /$ $\mathrm{mm}^{3}$; ESR $7 \mathrm{~mm}$ in $1 \mathrm{hr}$; blood urea, $11 \cdot 1 \mathrm{mmol} / 1$ (normal 2:5-7.1); serum electrolytes, normal.

X-ray of the chest showed an enlarged heart with dilatation of the ascending aorta with unfolding of the arch; clear lung fields with emphysematous changes; extensive Paget's disease of the shoulder girdles. X-ray of the skull (lateral) showed gross Paget's disease with flattening of the base, and basilar invagination (Fig. 4). 


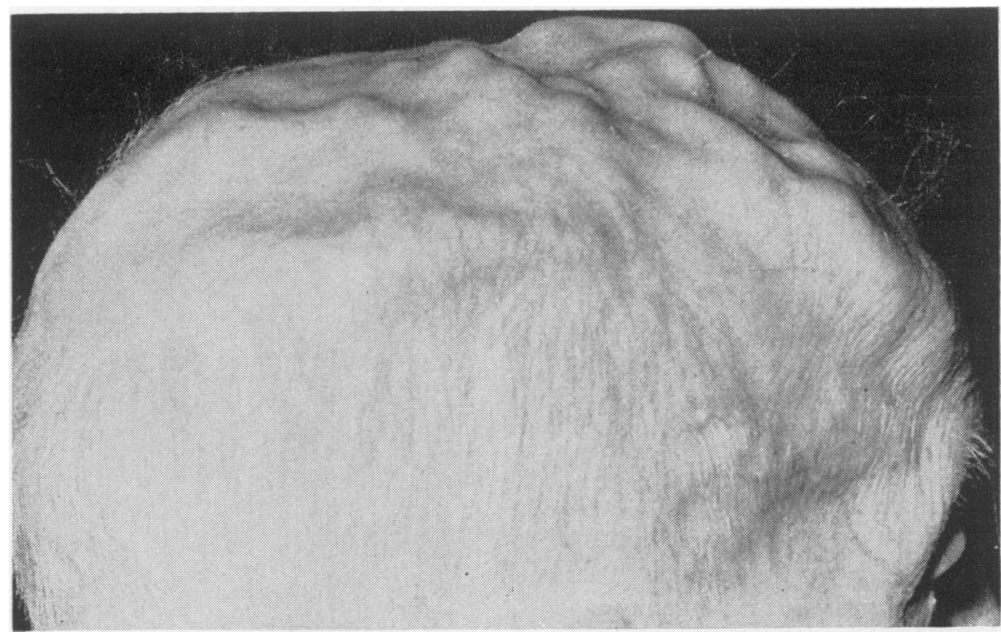

FIG. 3. Case no. 1. A close lateral view of the head showing gross wavy pattern of the head (skull).

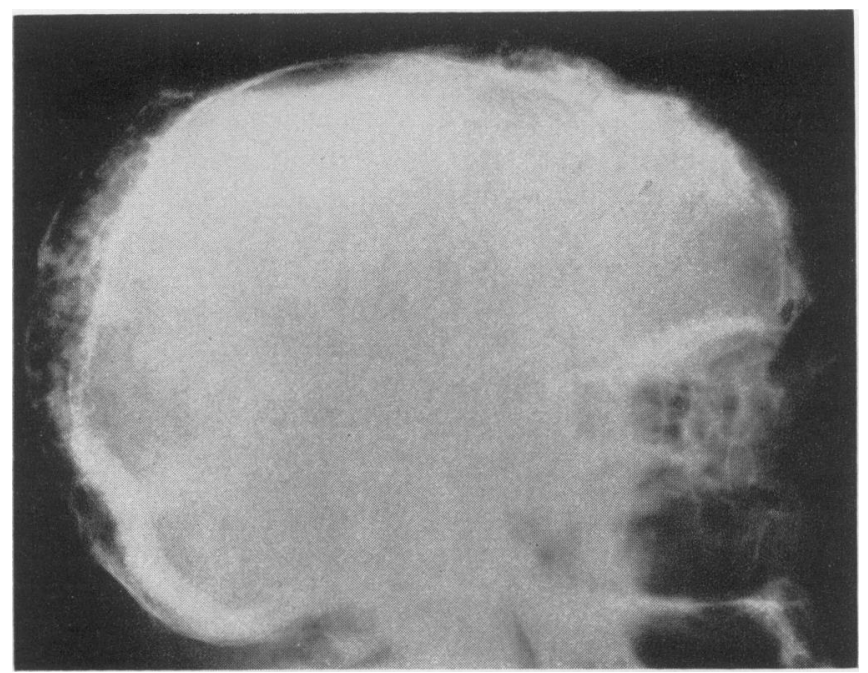

FIG. 4. Case no. 1. X-ray of the skull (lateral view) showing gross changes due to Paget's disease, i.e. thickening of the outer table, irregular thickening of the vault and patchy areas of sclerosis and translucency, etc.

$\mathrm{X}$-ray of the lumbar spine and pelvis showed gross changes due to Paget's disease, with softening of the pelvis which had led to protrusio acetabuli (Fig. 5).

$\mathrm{X}$-ray of the femora showed gross Paget's disease and an old fracture of the left lower femur.

ECG revealed left axis deviation with atrial fibrillation. Serum calcium and phosphorus, normal; serum alkaline phosphatase, $113 \mathrm{KAu} / 100 \mathrm{ml}$ (normal, 3-12 KAu); serum bilirubin, serum proteins, SGOT, SGPT were all normal; total hydroxyproline excretion in the urine $130 \mathrm{mg} / 24 \mathrm{hr}$ (high); hydroxyproline/creatinine ratio, $\mathbf{0 . 2 2 8}$ (normal, $<0.024$ ).

Despite the patient's advanced age, treatment with calcitonin was under consideration because of his bone pain.

\section{Case 2}

An 80-year-old female gave a history of increasing deafness over the last 8 years (severe deafness made it difficult to obtain a good history from her), and she had noticed irregularity of her head (Fig. 6a, b) for at 


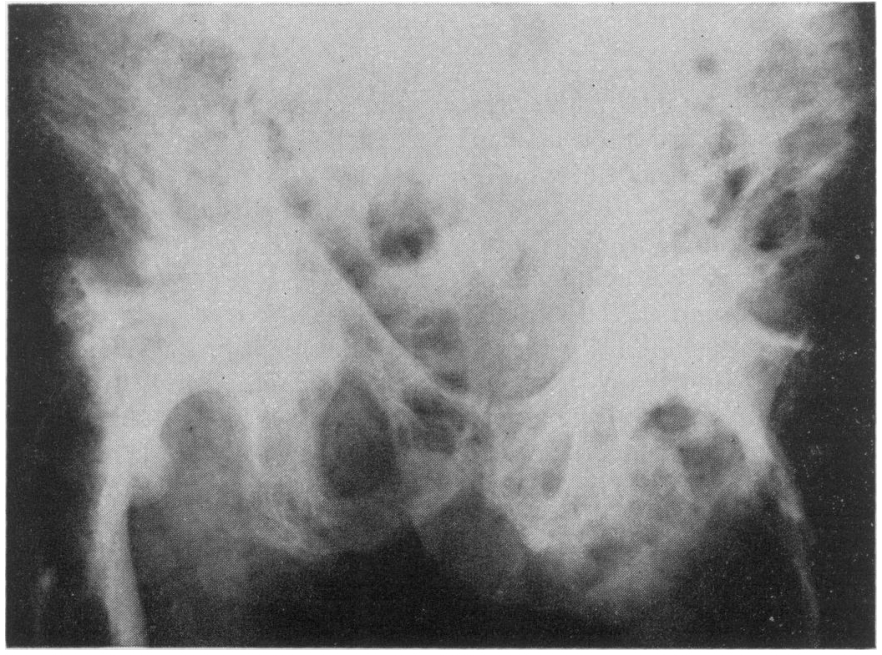

(2)

FIG. 5. Case no. 1. X-ray of the pelvis showing extensive changes due to Paget's disease involving the whole pelvis. The trabecular pattern is very coarse. The pelvis is compressed leading to protrusio acetabuli and a triradiate deformity. The upper part of both femora is also involved.

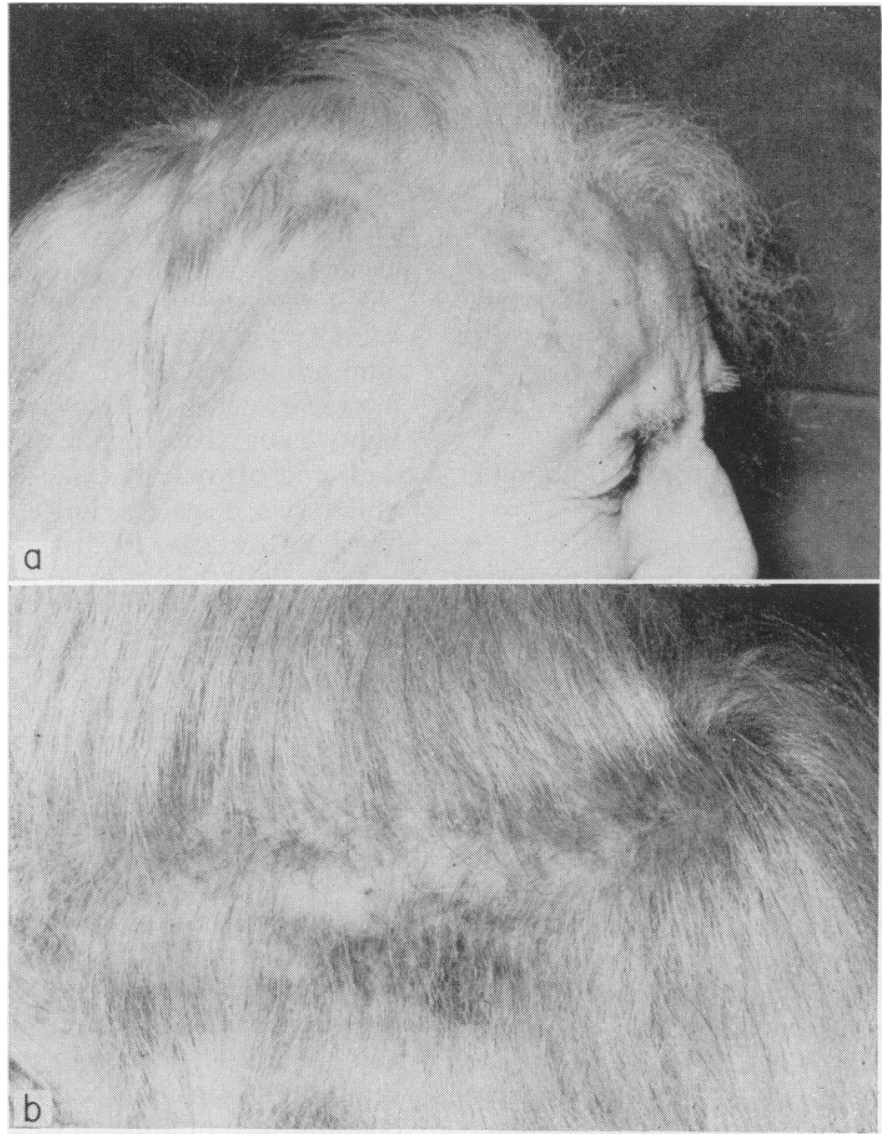

Fig. 6a, b. Case no. 2. Lateral views of the head showing wavy shapc of the head (skull). There is marked irregularity involving almost the whole of the skull but, unfortunately, in the picture it is mostly obscured by the patient's long hair. The irregularity is detected much more satisfactorily on palpation of the head (skull). 
least 10 years. She was partially sighted. She was ambulant but needed minimal supervision because of impaired vision. She had had a cholecystectomy 7 years previously.

The patient was a pleasant, frail, elderly lady with no obvious anaemia. There was no evidence of mental impairment, and she scored $10 / 10$ on the mental status questionnaire test. Her blood pressure was $200 / 90 \mathrm{mmHg}$. Heart examination revealed cardiomegaly with soft systolic murmur in the apical area. Heart rate was $60 / \mathrm{min}$ (regular). There was evidence of moderate congestive cardiac failure with raised jugulo-venous pressure and peripheral oedema. Chest examination did not show any significant abnormalities. Abdominal examination revealed no other abnormalities. Palpation of the skull revealed gross irregularities with warmth of the scalp (due to increased blood flow). There was no bruit heard on auscultation. Apart from marked deafness and visual impairment there was no other neurological deficit.

\section{Investigations}

Haemoglobin $13 \mathrm{~g} / \mathrm{dl}$; white cell count, $6000 / \mathrm{mm}^{3}$; PCV, $42 \%$; MCHC, $31 \%$; ESR, $13 \mathrm{~mm}$ in $1 \mathrm{hr}$.

$\mathrm{X}$-ray of the chest revealed an enlarged heart with left ventricular preponderance; lung fields showed bronchitic changes.

$\mathrm{X}$-ray of the skull and pelvis showed gross changes from Paget's disease.

Serum alkaline phosphatase, $34 \cdot 2$ KAu (normal, 3-12 KAu); total hydroxyproline excretion in the urine, $0.634 \mathrm{mmol} / 24 \mathrm{hr}$ (high); hydroxyproline/ creatinine ratio, 0.131 (high); total protein $6 \mathrm{~g} \%$; albumin $2.9 \mathrm{~g} \%$; globulin $3.1 \mathrm{~g} \%$.

Electrophoresis, normal except for low albumin.

\section{Case 3}

An 87-year-old male attending the out-patients' clinic for the last few years with recurrent pain in the joints, particularly the right hip, having sustained a subcapital fracture to the left femur following a fall in 1971, had no evidence of Paget's disease in the femur. However, at his last attendance he complained of frequent headaches which had recently become unbearable with little relief from ordinary analgesics. He denied having had any episodes of dizziness. He gave a history of suffering from chronic bronchitis and 'angina'. He walked quite well with the help of zimmer aid.

The patient was quite rational and scored $10 / 10$ on the mental status questionnaire test. He had slight pallor of the mucus membranes. Examination of the cardio-vascular system revealed his blood pressure to be $160 / 90 \mathrm{mmHg}$; heart rate $72 / \mathrm{min}$ (regular), but no significant cardiomegaly. There was no evidence of congestive cardiac failure. Examination of the chest was non-contributory. No neurological deficit was detected (including deafness). The movements of the hip joints were painful and somewhat restricted. Inspection and palpation revealed marked corrugation of the skull (Figs 7 and 8).

\section{Investigations}

Haemoglobin, $11.7 \mathrm{~g} / \mathrm{dl}$; white cell count, $9300 /$ $\mathrm{mm}^{3}$; ESR, $50 \mathrm{~mm}$ in $1 \mathrm{hr}$; blood urea, $9.8 \mathrm{mmol} / 1$ (normal, $2 \cdot 5-7 \cdot 1)$; serum electrolytes, normal.

$\mathrm{X}$-ray of the chest showed a slightly enlarged heart; chronic bronchitic and emphysematous changes.

$\mathrm{X}$-ray of the skull showed thickening of the skull vault associated with osteoporosis due to Paget's disease.

X-ray of the pelvis, hips and femora showed osteoarthritic changes of the hip joints only; no evidence of Paget's disease.

ECG revealed sinus rhythm, left axis deviation and ischaemic changes.

Serum calcium, $2.43 \mathrm{mmol} / 1$ (normal); serum phosphorus, $0.83 \mathrm{mmol} / 1$ (normal); serum alkaline phosphatase, 20.6 KAu (normal, 3-12 KAu); urinary hydroxyproline, 0.44 (normal, $0.02 \mathrm{mmo} / \mathrm{l}$ ).

This patient had radiological evidence of Paget's disease affecting only the skull bones, whereas patient no. 1 had had multiple bones involved and patient no. 2 had involvement of the skull and pelvis

\section{Incidence}

A 1-year survey of Paget's disease was carried out: in a London hospital which revealed that out of the total admission of (all diseases) 5000 cases, eighty showed evidence of Paget's disease of bone. Of those eighty cases, sixteen patients had involvement of the skull and of these, six had corrugation of the skull which was detected clinically (on palpation only) (Das and Kataria, 1975b).

TABLE 1. Findings from a 1-year survey of Paget's Disease at a London hospital

\begin{tabular}{lc}
\hline Total admissions & $\begin{array}{c}\text { Per year } \\
\text { (average) }\end{array}$ \\
\hline Of all diseases & 5000 \\
Paget's disease & $80(1: 70)$ \\
Skull involvement & $16(20 \%)$ \\
Corrugated skull & $6(7.5 \%)$ \\
\hline
\end{tabular}

In the hospitals in the Kirklees Area Health Authority, out of fifty cases which showed evidence of Paget's disease of bone, three patients had corrugation of the skull detected clinically (by inspection and palpation). The incidence of corrugation of the skull in Paget's disease of bone appears to be about one in fifteen of affected cases. This, of course, does not take into consideration cases which are asymptomatic (at least $20 \%$ ), and only diagnosed incidentally 

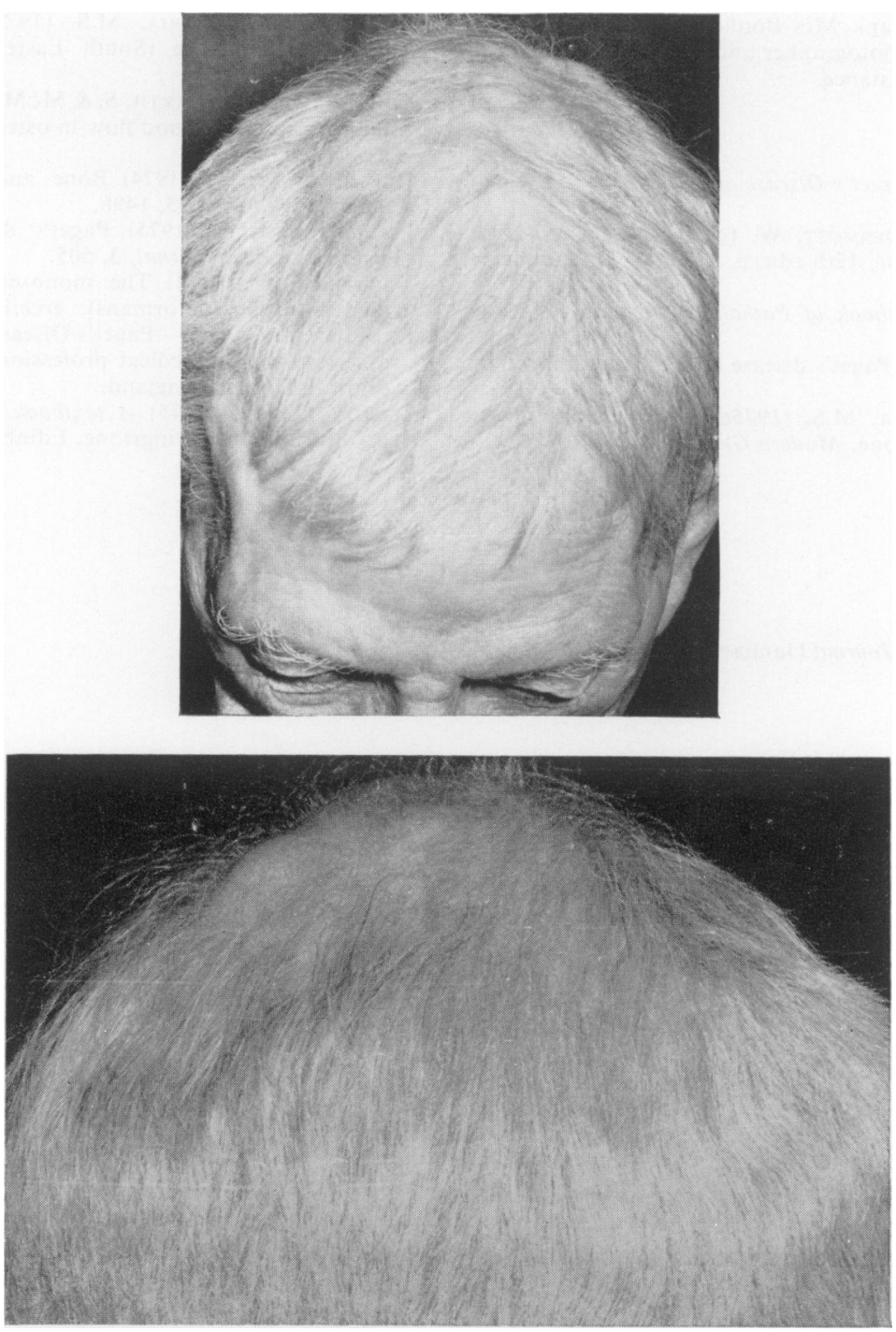

Figs. 7 and 8. Case no 3. Photographs (front and lateral views) showing corrugation of the head (skull) partly obscured by the patient's hair.

when radiological examination is carried out for other reasons, or who have been found to have an elevated serum alkaline phosphatase (without obvious cause).

\section{Comment}

Corrugation of the skull is an unusual feature of Paget's disease of bone, but when present can be regarded as a useful physical sign. There does not appear to be a description of this feature in the medical text books. It is suggested that inspection and palpation of the skull may be helpful in diagnosing this disease. When corrugation is detected radiological examination of the skull and pelvis should be carried out. This, in association with high estimations of serum alkaline phosphatase and urinary hydroxyproline, should confirm the diagnosis.

Nowadays, effective therapy with calcitonin is claimed not only to arrest but also to reverse the disease process.

\section{Acknowledgments}

We wish to thank Dr D. S. Harling, Dr Stonawski and Dr Haugie for allowing us to use their cases and Dr G. Annan for his help in preparing the manuscript. 
We also wish to thank Mrs Bonfield, medical secretary, Mr Morris, medical photographer and Mrs Calvert, medical librarian, for their assistance.

\section{References}

Barry, H.C. (1969) Paget's Disease of Bone. E. \& S. Livingstone Ltd, London.

Beeson, P.B. \& McDermott, W. (eds) (1968) Cecil-Loeb Textbook of Medicine, 12th edn, p. 1385. W. B. Saunders, Philadelphia.

Boyd, W. (1970) Textbook of Pathology, 8th edn, p. 1314. Kimpton, London.

Collins, D.H. (1956) Paget's disease of the bone. Lancet, ii, 51.

DAS, S.K. \& Kataria, M.S. (1975a) Corrugated skull in Paget's disease of bone. Modern Geriatrics, 5, 25.
Das, S.K. \& Kataria, M.S. (1975b) British Geriatrics Society Conference (South Eastern Region). Age and Ageing, 4, 63.

Edholm, O.G., Howarth, S. \& McMichael, I. (1945) Heart failure and bone blood flow in osteitis deformans. Clinical Science, 5, 249.

Hodkinson, H.M. (1974) Bone and joint disease in the elderly. Medicine, 25, 1496.

Leading Article (1975) Paget's disease and calcitonin. British Medical Journal, 3, 505.

Newton, F.C. (1924) The mono-osteitic type of Paget's disease (osteitis deformans). Archives of Surgery, 8, 24.

Radiological Atlas-Paget's Disease of Bone, produced as a service to the medical profession by Armour Pharmaceutical Co. Ltd, England.

Sutton, D. (ed.) (1975) A textbook of Radiology, 2nd edn, p. 78. Churchill Livingstone, Edinburgh.

Postgraduate Medical Journal (January 1977) 53, 46-48.

\section{Myxoedema coma presenting in status epilepticus}

\author{
K. L. Woods \\ M.B., M.R.C.P.
}

\author{
G. K. T. Holmes \\ M.D., M.R.C.P.
}

Selly Oak Hospital, Birmingham

\begin{abstract}
Summary
A 71-year-old woman with myxoedema coma presenting in status epilepticus is reported. Although this complication of myxoedema coma is considered to be fatal the patient described responded dramatically to treatment and remains in good health.
\end{abstract}

\section{Introduction}

Although still rare, myxoedema with coma has been recognized with increasing frequency in recent years. It is probable that many cases arise as a late consequence of surgical or isotope treatment for thyrotoxicosis. The mortality remains high and prompt diagnosis is vital. In the few instances where fits accompanied coma, a fatal outcome has been almost invariable. The case is reported of a severely hypothyroid patient who presented in status epilepticus and responded well to treatment.

\section{Case report}

A 71-year-old woman was seen by her general practitioner one evening because of confusion and disorientation which had developed over the previous few hours. She was noted to be cold, pale and apparently suffering from acute bronchitis. There was no past history of note. She refused admission and was given $25 \mathrm{mg}$ of chlorpromazine and started on ampicillin. Two hours later she began to have grand mal convulsions and was urgently transferred to hospital.

On arrival she was cyanosed, deeply unconscious and in status epilepticus. Her colour improved after intubation, removal of vomit from the trachea, and assisted ventilation. The fits were controlled with diazepam $10 \mathrm{mg}$ intravenously. Thereafter, adequate spontaneous respiration was maintained.

Examination revealed puffiness of the face with oedema of the eyelids and a dry, coarse skin; the hair was scanty. Her rectal temperature was $31^{\circ} \mathrm{C}$. The blood pressure was initially unrecordable. There were no focal signs in the nervous system; the pupils were small and equal, and the limbs hypotonic with absent reflexes. Throughout both lungs coarse crepitations could be heard. The abdomen was cold to the touch but otherwise unremarkable. 